\title{
Visual discrimination of motion: Stimulus relationships at threshold and the question of luminance-time reciprocity*
}

\author{
DAVID C. HENDERSON \\ Columbia University, New York, New York 10027
}

\begin{abstract}
Quantitative descriptions, derived from an earlier empirical study, of threshold relationships among the principal stimulus variables for motion discrimination are presented, with emphasis on evaluating the influence of stimulus energy content. The present findings are compared with those of R. H. Brown $(1955,1957,1958)$; who leported the applicability of luminance-time reciprocity to motion threshold for exposures shorter than 0.1 sec. It is demonstrated that such reciprocity is atypical of motion threshold. and that it represents limitations imposed by the requirements of target visibility rather than by motion discrimination itself. It is also shown that Brown's data are predictable from the present equations.
\end{abstract}

In a series of landmark studies, R. H. Brown (1955. $1957,1958)$ reported data which seemed to demonstrate the applicability of Bloch's law (of intensity-time reciprocity) to motion discrimination and, therefore. to provide evidence for the existence of an energy-based mechanism subserving the discrimination. If, as Brown concluded, motion is signaled upon the accumulation of a critical energy quantity, then the fundamental parameters of motion-velocity and its two defining variables. time and distance of transit-influence the discrimination only by influencing the amount of energy delivered. If, on the other hand, motion perception depends primarily on neural information about the sequential stimulation of successive retinal loci (i.e.. temporal and spatial information), then energy associated with the stimulus event would be important only insofar as it influences the preservation and transmission of this information.

Brown measured the luminance required to permit correct report of direction of movement of a small target. In general. it may be said that, given movement durations of less than $0.1 \mathrm{sec}$. Brown's data seem to reflect energy constancy (luminance-time reciprocity) at threshold, at least for a certain area within the coordinate space of the relevant stimulus dimensions. Brown's findings and conclusions seem to have been generally accepted. and there have been few subsequent studies characterized by a comparably analytical approach to the stimulus whose results might be useful in evaluating or corroborating them.

On the basis of data reported previously (Henderson. 1971), this paper will attempt to demonstrate (1) that such energy limits are far from representative of the

*This work was supported by PHS Research Grants 5R01 EY 0039103 and EY 0037504 from the National Eye Institute. by the Institutional Scientific Research Pool of Columbia University, and by Contract N00014-67-A-0108-0009 between Columbia University and the Office of Naval Research. This paper is Technical Report No. 12 of the latter contract. thresholds of movement discrimination, (2) that even those circumstances under which energy constancy applies reflect not the requirements of motion discrimination but rather those of simple target visibility, (3) that for any but extremely dim stimuli, threshold is, in fact, determined by an inverse relationship between time and distance rather than between time and intensity, and particularly (4) that Brown's data are consistent with. and indeed almost entirely predictable from, such a relationship.

Evaluation of the minimum stimulus requirements of the perceptual mechanism subserving motion discrimination seems of particular importance in light of recent findings of processing at low levels in the visual system (Barlow \& Hill, 1963; Barlow. Hill. \& Levick. 1964; Oyster, 1968). The relationships governing these requirements demarcate the constraints within which models of neural detecting mechanisms must be constructed. At issue is the question of which aspects of the physical dimensions constituting the moving stimulus are most influential in contributing to a perceptual effect and in what way liminal values on these dimensions are related. Brown (1957) concluded from his findings that for exposures shorter than about $0.1 \mathrm{sec}$ and for moderate speeds. the discrimination of motion "involves a single sensory event controlled by the initial photochemical activity in the retina." This notion has superficial appeal in light of the subsequent discovery of motion-selective ganglion activity (e.g.. Barlow \& Hill, 1963). but on further consideration it taxes the imagination somewhat to devise a hypothetical motion detector which could be indifferent to the elemental constituents of velocity. i.e.. time and distance. except. in Brown's words. "in the sense of providing for the supply of energy." The disconcerting implication of this assertion is that within certain. but fairly generous. limits, stimulus motion will be detectable regardless of its velocity or the time or distance of travel. so long as the target is sufficiently brightt. 


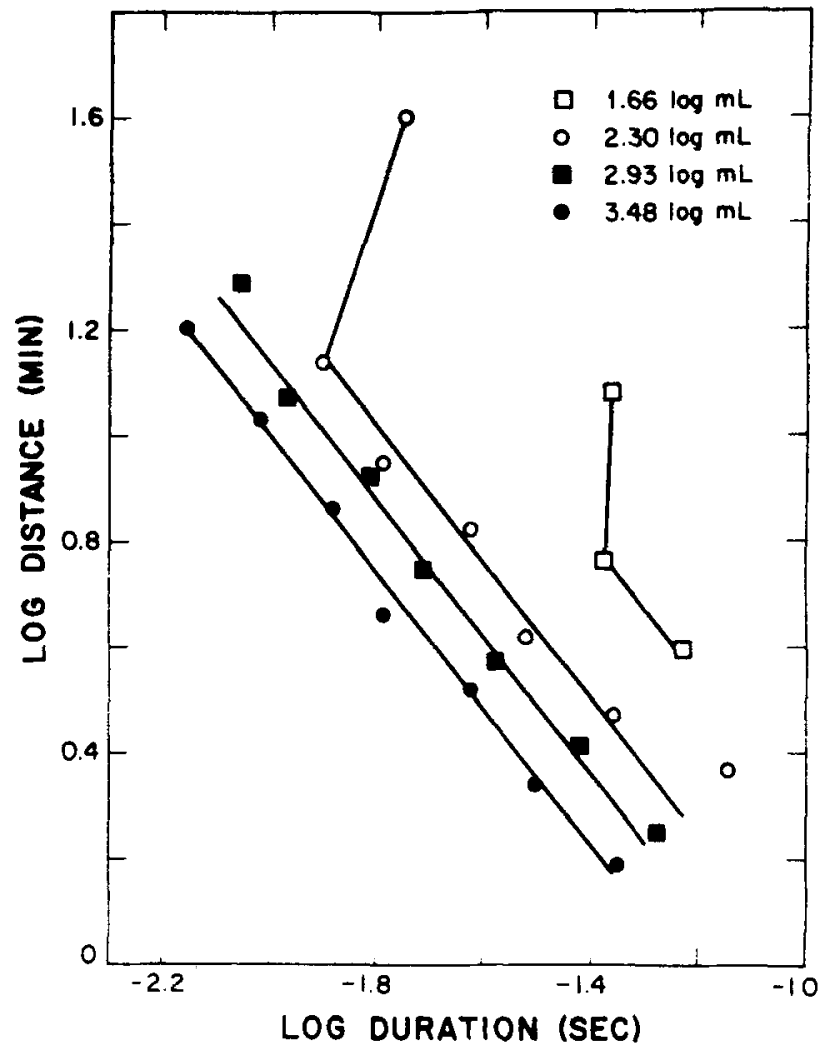

Fig. 1. Threshold distance for motion discrimination as a function of threshold duration, for four values of target luminance.

The threshold curves of Henderson (1971) show a fairly abrupt discontinuity at exposure durations of about $0.1 \mathrm{sec}$, segregating different empirical functions for greater and smaller durations. Cohen and Bonnet (1972) report finding constancy of motion extent at threshold for the longer durations (and slower speeds) and suggest that the long-exposure data of Henderson reflect this constancy. The present paper will concern itself only with exposure durations of less than roughly $0.1 \mathrm{sec}$. and all unqualified statements should be interpreted as implying this restriction. These short durations, however, do not represent atypical conditions for movement discrimination. It will be seen that for all velocities greater than a relatively slow speed (about $1 \mathrm{deg} / \mathrm{sec}$ ), motion threshold is achieved with such short exposures. It is also important to note that as is true of any discrimination, the perceptual event at "motion threshold" is the same regardless of by which relevant variable-velocity. time, distance. or luminance-it is being measured. and that when any one of these stimulus dimensions is statistically at threshold so, necessarily. are all the others. regardless of which is termed dependent. independent, or parametric.

The procedure and stimulus display employed are described in detail in Henderson (1971). Briefly. a 1.4-min-diam foveal target appeared abruptly in motion against a dimmer background. moved for some variable distance and duration at some constant velocity: and disappeared abruptly while still in motion. Movement was always in the vertical axis, and the $S$ was asked to report its direction (up or down) if motion was discriminable. It is presumed that the general form of threshold relationships obtained with the present stimulus display is valid for brief movements of any small luminous target.

\section{PRESENT FINDINGS}

\section{A Time-Distance Tradeoff}

The findings of Henderson (1971) indicated that. given $t<0.1 \mathrm{sec}$. threshold for movement discrimination is relatively insensitive to target luminance but is. rather, dependent under most conditions principally on time and distance of transit, according to a "tradeoff" relationship between these two variables. For a given luminance, this relationship is expressed by

$$
\mathrm{dt}^{\mathrm{m}}=\mathrm{C}
$$

where $t$ and $d$ are time and distance of movement (variables which determine threshold velocity) and $\mathrm{C}$ is a constant. The value of the exponent $m$ varies only with background luminance and was found to be less than or equal to one for the backgrounds employed.

A family of empirical functions exhibiting the tradeoff relation expressed by Eq. 1 is shown in Fig. 1. The four functions represent four target luminances incremented in equal logarithmic steps, higher values of $\mathrm{L}$ yielding curves positioned lower on the ordinate. The curves are parallel, implying independence of the relationship between $\mathrm{d}$ and $\mathrm{t}$ from stimulus luminance. which operates on the constant. C. in Eq. 1. For the background luminance $(-.22 \log \mathrm{mL})$ prevailing for the experimental conditions of Fig. 1 . both the $S$ shown and another $S$ yielded a value close to unity for the exponent. m. The Fig. 1 data, then. portray nearly a simple reciprocity between time and distance at threshold for movement discrimination. although for a brighter background $m$ was found to be about $1 / 2$. For the two lower target luminances in Fig. 1. the threshold functions break rather sharply into roughly vertical upper limbs. representing. at least for the $1.66 \log \mathrm{mL}$ function, duration constancy across velocity (as d increases). For these conditions, then. duration assumes almost single-handedly the limiting role in the discrimination, and these limbs reflect approximately constant energy. Such energy or duration constancy. however. is hardly representative of movement discrimination threshold for most combinations of the principal variables. time. distance. and luminance. as Fig. 1 clearly shows.

The term "time-distance tradeoff" has been used to describe the relationship seen in Fig. 1 since it connotes accurately the way these two variables interact to 
Fig. 2. Threshold distance for motion discrimination as a function of target luminance for a fixed velocity $(57 \mathrm{~min} / \mathrm{sec})$.

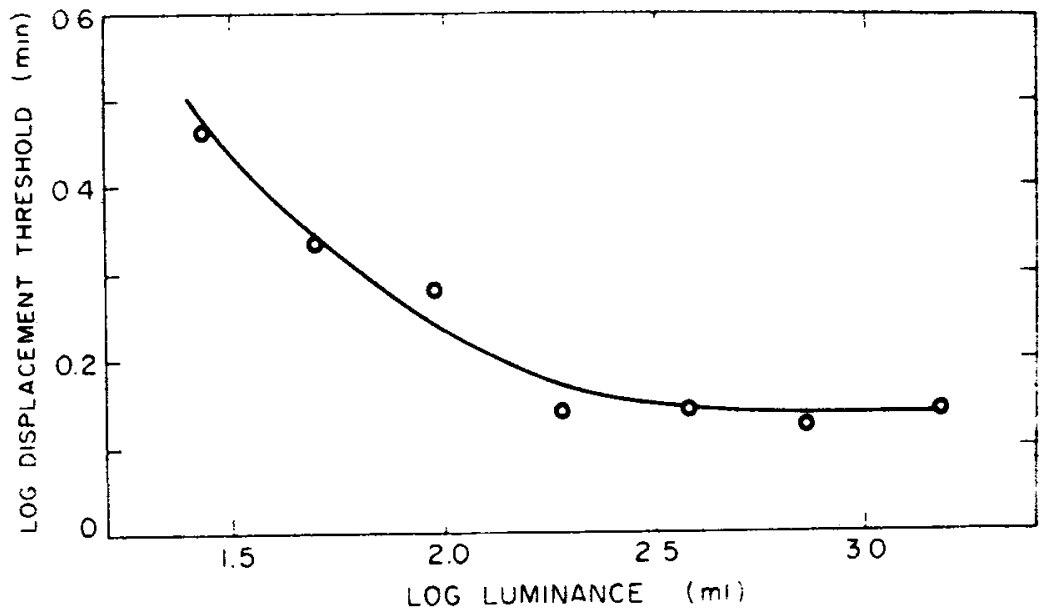

establish a threshold effect. A statistically subthreshold movement may be brought to threshold by increasing either the time or distance of movement. Or, given a threshold movement, the threshold effect may be sustained when either time or distance is decreased by increasing the other in the same proportion-that is, by trading time for distance. or vice versa. In the earlier paper (Henderson, 1971), it was suggested that this reciprocal relationship between liminal values on these two dimensions might implicate the mechanisms of spatial and temporal integration. But. in diametric opposition to R. H. Brown's assumptions, it was hypothesized that movement discrimination is obstructed rather than facilitated by these mechanisms. It was supposed that discrimination of movement might depend on the concurrent discriminability of the temporal and spatial distinctiveness of retinal events associated with the two endpoints of the motion path, and that such discriminability should be opposed by the retina's tendency to integrate over each of these two dimensions. There is evidence (Bouman \& van den Brink, 1952; Graham \& Margaria. 1935: Sperling \& Jolliffe. $1965)$ that the maximum value of either dimension over which complete integration may occur is inversely related to the prevailing value of the other.

\section{Luminance Effects}

Target luminance is embodied as a parameter in the constant. C, in Eq. 1. but its influence is described by a decay function which leaves $C$ virtually unchanged for luminances exceeding an intermediate value. Figure 2 shows the empirical relationship between threshold distance of movement and luminance for a fixed velocity: an inverted function may be extracted for the relationship between $t$ and $L$, since $t$ and $d$ are reciprocally related. For any fixed velocity; the relationship manifest in Fig. 2 may be described by

$$
d=k_{1}\left(L+k_{2}\right)^{-c}+k_{3}
$$

where $\mathrm{k}_{1}, \mathrm{k}_{2}, \mathrm{k}_{3}$, and $\mathrm{c}$ are constants. Faster velocities yield parallel curves displaced higher on the ordinate and larger values of $k_{1}$ and $k_{3}$. The steepness of the descending portion of the curve is exaggerated by wider log-unit spacing on the Fig. 2 ordinate than on the abscissa; in fact. luminance exercises fairly weak leverage on threshold even for this portion. and none at all for higher luminances.

The relationship expressed by Eq. 2 contrasts with the requirement of almost any energy-based model that there be a simple reciprocity between threshold distance and target luminance. assuming distances short enough to permit complete spatio-temporal integration: that is, for $\mathrm{V}$ $=V_{i}$, assuming $L t=k$ requires that $d=k V_{i} L$, since $d=V t$. A second way in which the data fail to reflect energy constancy is evident in Eq. 1. which applies when target luminance is fixed. Constancy of the energy product $\mathbf{L t}$ would demand constant duration for a given luminance. but Eq. 1 shows that threshold requirements permitted variation in such duration. Threshold energy constancy was obtained, however, for certain restricted and aty pical stimulus conditions (vertical limbs. Fig. 1), principally combinations of low target luminance and high velocity. which will be discussed below.

\section{General Equation}

The constant. C. in Eq. 1 may be evaluated using Eq. 2 in order to derive the general expression capable of describing the threshold stimulus in terms of any of the four principal stimulus variables: 


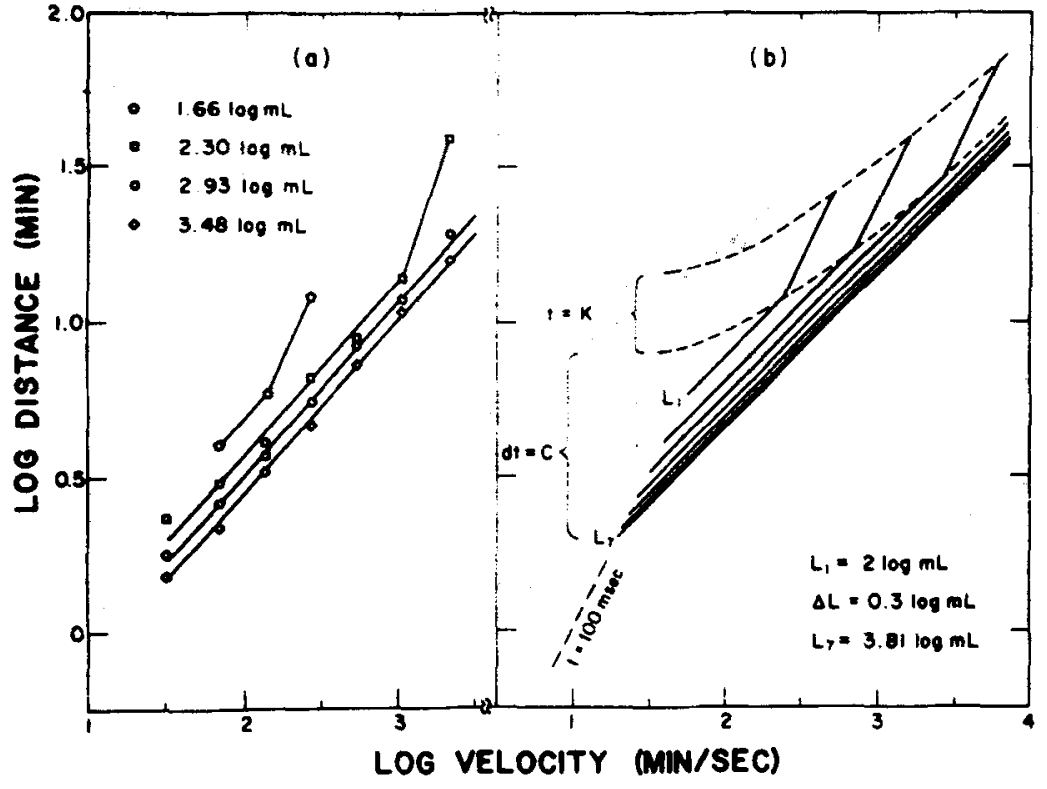

Fig. 3. Threshold distance as a function of velocity for different fixed values of target luminance. (a) empirical data: (b) theoretical curves generated by Eq. 4 .

$$
\mathrm{dt}^{\mathrm{m}}=\left[\mathrm{K}_{1}\left(\mathrm{~L}+\mathrm{K}_{2}\right)^{-\mathrm{c}}+\mathrm{K}_{3}\right]^{\mathrm{m}+1}
$$

where $K_{1}$ and $K_{2}$ are constants derived from $k_{1}$ and $k_{2}$ of Eq. 2. but, unlike them, are independent of velocity. By making the proper substitutions from the defining equation of velocity. $V=d / t$, it is possible to solve $E q .3$ for the relationship at threshold between any two of the variables $\mathrm{V}, \mathrm{d}, \mathrm{t}$. and $\mathrm{L}$. with the remaining free variable as a parameter. As will be seen, however, a complete picture of such threshold relationships requires that functions generated by Eq. 3 yield. under certain restricted conditions, to functions representing approximate time-luminance reciprocity, as do two of the functions of Fig. 1.

Figures $3 \mathrm{a}$ and $3 \mathrm{~b}$ show, respectively, empirical data (the same data plotted in Fig. 1) and theoretical curves generated by a computer from the solution of Eq. 3 for threshold distance as a function of velocity, with luminance as a parameter:

$$
d=\left[K_{1}\left(L+K_{2}\right)+K_{3}\right] V^{\frac{m}{m+1}}
$$

Equation 4 constants were estimated roughly from the data of a $S$ other than the one represented in Fig. 3a. In Fig. 3b, Eq. 4 applies below the lower dotted line, which associates points of equal energy across the different luminance functions. All points on the unit-slope limbs extending above the dotted line represent this single energy content and reflect, for each luminance function, the same constancy of duration seen for the two lowest luminances in Fig. 1. The rationale for this bilinear fit will be developed below. In Fig. 3a, no data could be obtained for the two lower luminances at velocities higher than those associated with the final point on each function, since faster speeds distributed the stimulus energy so thinly that the target could not even be seen. Similarly, points associated by the upper dashed line in Fig. $3 b$ represent, for each luminance, the maximum velocity for which the stimulus should be visible.

\section{Energy Constancy}

Figure 4 demonstrates more clearly the variation in stimulus energy content which is typical of motion threshold as well as the conditions under which energy constancy was obtained. Log energy and log duration (since the two are proportional) at threshold are plotted for a single luminance as a function of $\log$ velocity. These data represent a brighter background than do those of Fig. 1 and are characterized by a value of approximately $1 / 2$ for the exponent, $m$, in Eqs. 1 and 3 . The flat dashed line is a hypothetical curve representing threshold energy for simple visibility of the moving target-that is, conditions permitting detection of the stimulus but not discrimination of its movement. At least for constant displacements, absolute visibility of moving targets has been shown to manifest energy constancy at threshold (R. H. Brown. 1957; Bouman \& van den Brink. 1953). And R. H. Brown (1958) has shown. in addition, that for certain conditions, including low target luminances, visibility threshold describes a function parallel to and, in fact. almost coincidental with the threshold function for motion discrimination. Evidence which will be discussed later indicates. however. that the energy criterion for visibility increases when the distance of movement becomes large. The flat approximation is portrayed here for the sake of simplicity of the development and. in any case. 
represents no great distortion for the stimulus conditions of Fig. 4.

The descending portion of the motion discrimination function is describable by Eqs. 1 and 3, while the flat terminal portion represents constancy of duration and energy. It should be emphasized, however, that this function represents a fairly low luminance for the prevailing background, and that data obtained for higher luminances descend linearly and continuously for the entire velocity range tested and show no flat terminal limb. This limb represents the same effect manifest in Fig. 1 as vertical upper limbs of the two lower luminance functions. Moreover, even for the low luminances which exhibited it, this interval of duration constancy applied only for a small interval of the velocity range over which threshold durations were short enough to justify expectation of the involvement of integrating mechanisms. All such instances of constancy extended over no more than half a $\log$ unit of velocity due to the fact that a degree of spatio-temporal dispersion of energy was reached at a critical velocity beyond which the target could no longer be seen at all.

It is in this flat portion of the Fig. 4 data, then, that the present findings share with the data of Brown evidence of minimum-energy requirements at threshold. For these data points (these velocity-luminance combinations), however, it was the case that at the test stimulus value which yielded threshold for motion discrimination, the stimulus event itself was occasionally not even detected (50\% motion discrimination equaled roughly $85 \%$ absolute detection). In other words, it was almost the case that if a given stimulus event could be seen, its motion could be discriminated. Thus, under conditions which yielded the constant-energy limbs (and only for these conditions), motion threshold occurred at stimulus values only barely in excess of those necessary merely to sustain target visibility. The same effect is evident in the data of Brown (1958) and is portrayed in Fig. 4 by the close correspondence of the two functions over the velocity interval wherein energy constancy holds for motion discrimination.

Given exposure durations of less than $0.1 \mathrm{sec}$, then, motion threshold typically is described by the relationship expressed in Eq. 1 (descending portion of Fig. 4 data). For low target luminances, however, it appears that as the amount of energy exacted, more or less incidentally, by the time requirements of motion discrimination decreases with increasing velocity, it reaches a point beyond which those requirements would provide less energy than is necessary to sustain simple visibility. Beyond this point, visibility, with its associated demand for minimum energy, becomes the limiting factor for motion discrimination. For such conditions, time and distance are supplied in greater magnitude than Eq. 1 specifies as minimum requirements. By employing luminance as their threshold measure, Brown's procedures were selectively tuned to low-energy conditions, under which visibility

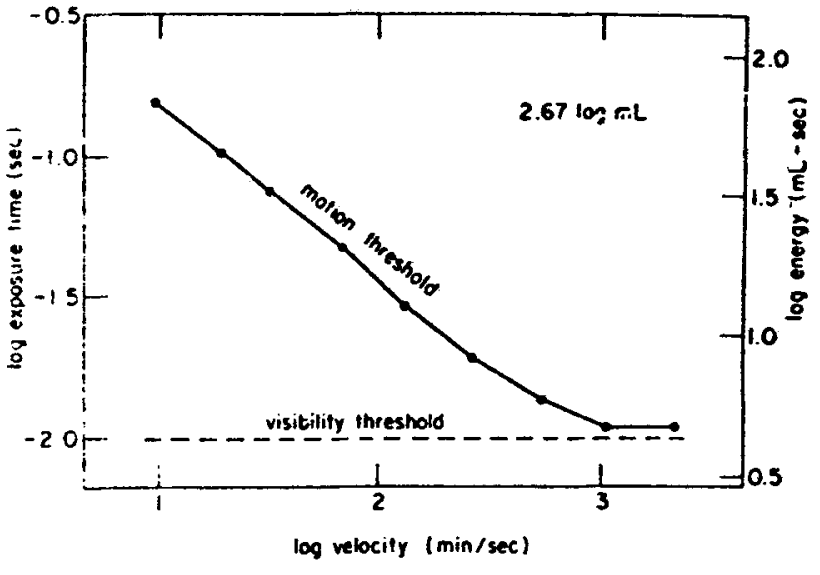

Fig. 4. Threshold energy and duration as a function of velocity for a single target luminance.

threshold assumes the limiting role in motion discrimination.

\section{R. H. BROWN'S FINDINGS}

\section{Energy Constancy}

R. H. Brown's 1957 and 1958 studies will be considered first here, since they involved tighter control of stimulus variables than did his first experiment in the series (1955) and are more readily amenable to analysis. Brown began with the hypothesis that "direct" movement perception depends on a single initial photochemical event, which in turn requires a critical energy input. Brown's experiments were designed to test this hypothesis by evaluating its implication that movement discrimination should show time-luminance reciprocity. Brown (1958) concludes from his data that "in order to produce a given visual effect, discrimination of motion in the present experiment, there is a reciprocal relation between stimulus luminance and exposure time," and suggests that the hypothesis has been verified.

For stimulus conditions comparable to those of the present author's research, Brown measured target luminance required to permit correct report of movement direction as a function of velocity for several fixed extents. Figure 5a presents a replotting of Brown`s 1957 group data, with functions for different distances of movement grouped here on common axes. The plot also includes Brown's measurements of absolute visibility for the moving stimulus, which were made when motion threshold could not be achieved even at high luminances. The linear ascending asymptotes have slopes of unity and represent reciprocity between stimulus luminance and exposure duration. A square on each data function denotes a duration of $0.1 \mathrm{sec}$, shorter durations being associated with higher velocities.

If those of Brown's motion thresholds which fall on the unit-slope asymptotes (filled circles) are segregated 


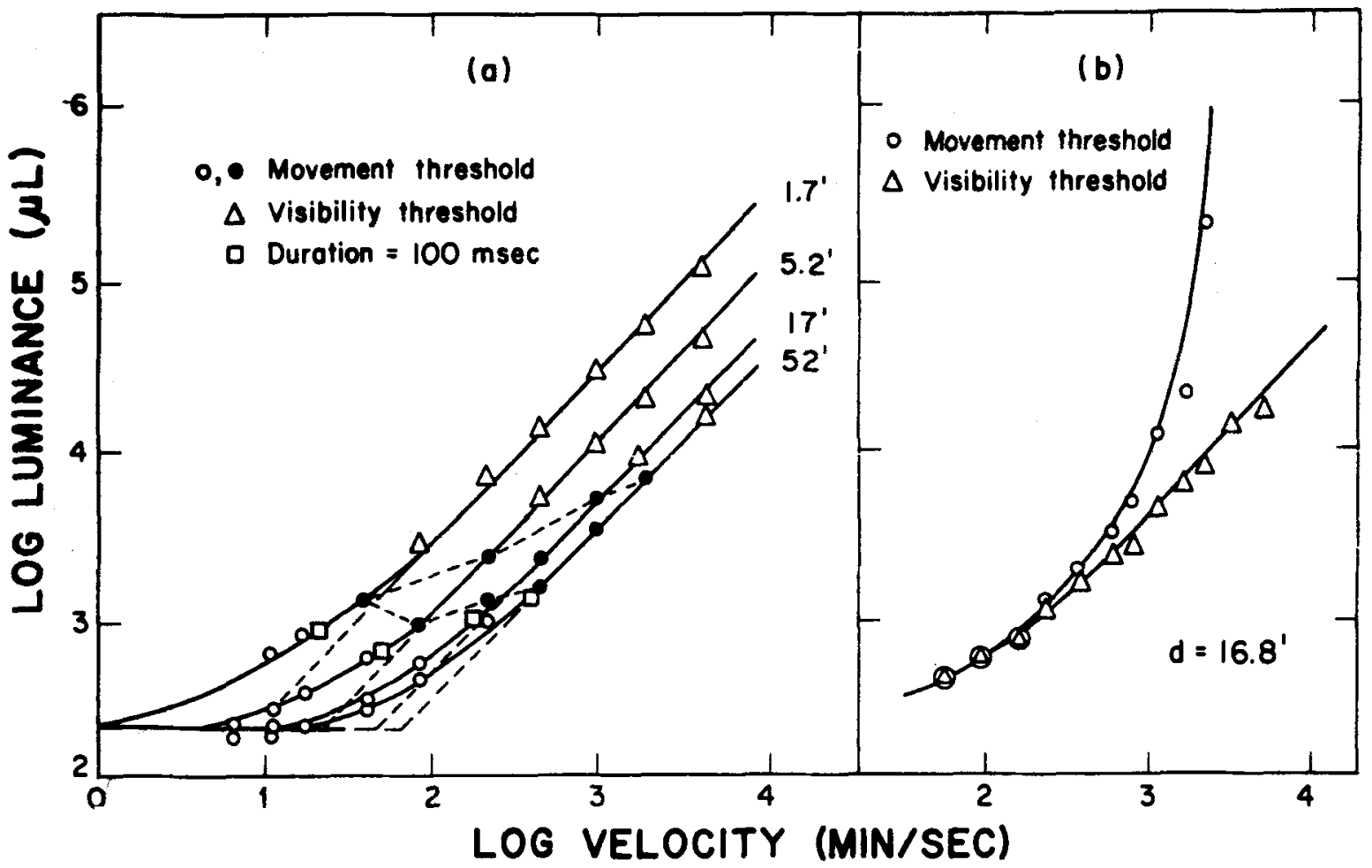

Fig. 5. Threshold luminance for motion discrimination and visibility as a function of velocity, for several fixed movement extents (Brown. 1957, 1958).

from those which do not (unfilled circles) and from the visibility thresholds (triangles), it is seen that the majority of the data points which in fact exhibit energy constancy are thresholds for simple target detection. A more complete picture of the two thresholds is given by Fig. $5 \mathrm{~b}$, which presents similar measurements made by Brown in 1958 for a single fairly long distance of movement. In this later study, however, both motion and visibility thresholds were determined for each velocity. It can be seen that the two functions are practically coincidental for lower velocities, but that the curve for motion threshold departs from the energy constancy asymptote, accelerating toward infinity at an asymptotic constant velocity. Motion at velocities above this critical speed could not be discriminated no matter how bright the target. A stationary line, or streak, was seen instead.

The same functions may be assumed to underlie the Fig. 5a data. The motion threshold data points of Fig. 5a may be visualized as accelerating rapidly upward from the highest such point on each extent function, with the asymptotic velocity being lower for smaller extents. This means that for each distance, there is a critical velocity at which motion discrimination becomes independent of luminance, and only visibility thresholds, which are well known to exhibit energy constancy, may be measured. Motion is discriminable at higher velocities. however. if the extent of motion is increased. This means that distance is to a much greater degree than energy a factor which determines the perceptibility of movement at a given speed.
There is a short interval in the Fig. $5 b$ data, between the divergence of the two curves and the point at which the motion-threshold curve approaches its vertical asymptote, wherein luminance does play an independent role. That is. increases in luminance can bring the stimulus from detection threshold to motion threshold. These data points, however, clearly do not reflect energy constancy. From the perspective of the coordinates of Fig. $5 \mathrm{~b}$, this is the same luminance effect reflected in Figs. 1, 2, and 3.

The virtual coincidence of the motion and visibility thresholds for lower velocities in Fig. $5 \mathrm{~b}$ also demonstrates a very important point about the general applicability of Brown's results: namely, that all motion-threshold data points in Fig. 5a which exhibit energy constancy (filled points) represent extremely dim targets, in fact, just barely above the threshold for absolute detection. These conditions, then, are hardly representative of the conditions under which motion is usually perceived.

\section{Predictions from Present Data}

The effect demonstrated by the convergence. with decreasing velocity. of the motion and visibility functions of Fig. $5 \mathrm{~b}$ is essentially the same as that demonstrated by the present author's data in Fig. 4 . although the two plots are in terms of different variables. This effect is that when the more generally 
Fig. 6. Theoretical curves relating threshold luminance for motion discrimination and velocity. with distance as a parameter.

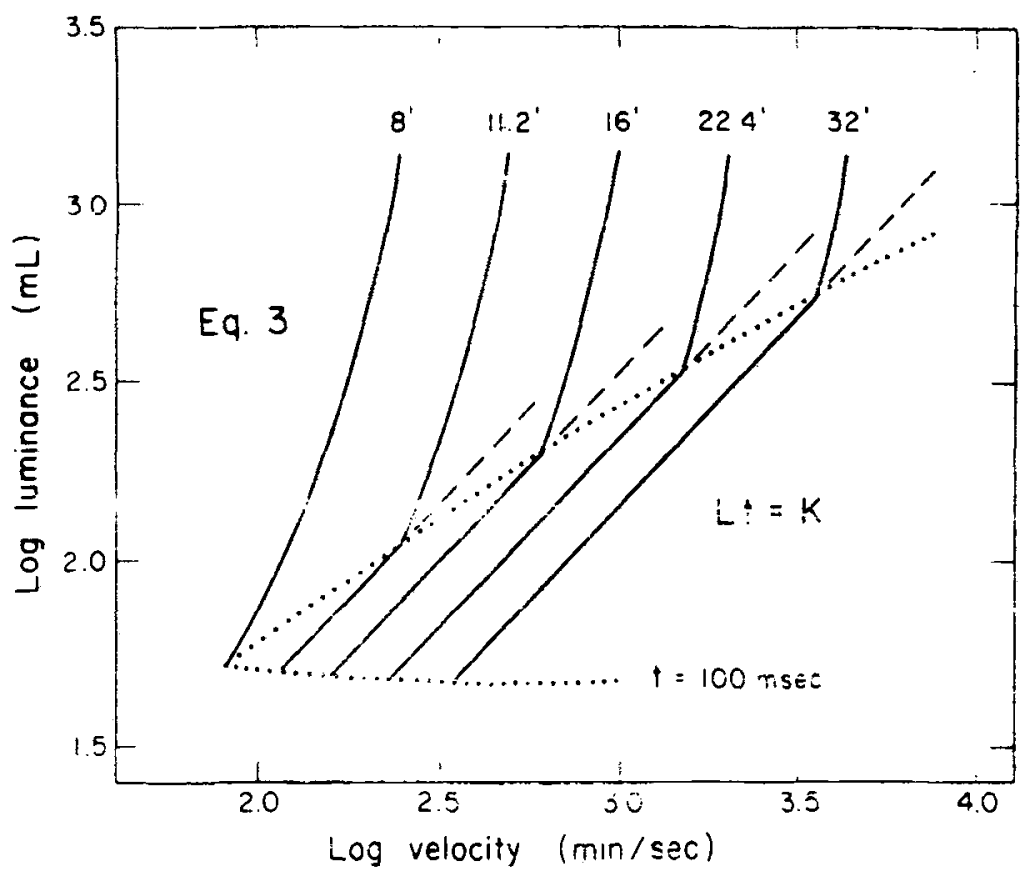

applicable threshold requirements. determined principally by time and distance. fail to exact sufficient energy to sustain stimulus visibility, visibility requirements assume the limiting role and their demand for minimum energy is transmitted to the motion threshold function. The departure of the motion threshold function in Fig. 5b (and the implied departures in $\mathrm{Fig}, 5 \mathrm{a}$ ) from the linear visibility functions. then. should reflect the operation of the threshold relations obtained for most conditions by the present author and described by Eq. 3 .

Figure 6 presents a set of computer-generated functions based on the appropriate solution of Eq. 3 . with distance as a parameter. As in Fig. $3 \mathrm{~b}$. these curvilinear functions have been made to yield to linear unit-slope functions. representing energy constancy. at points of equal energy across distance functions. This energy value was estimated from empirical measurements: estimates of Eq. 3 constants are the same as those used in generating the Fig. $3 \mathrm{~b}$ plot. All threshold functions are terminated at a lower velocity for which exposure time equals $0.1 \mathrm{sec}$ (lower dotted line).

It can be seen that the coordinate areas which represent energy constancy for motion threshold in Figs. $5 \mathrm{a}$ and 6 have a great deal in common. Both show that longer distances yield longer velocity intervals wherein motion threshold adheres to Lt reciprocity and that for shorter distances there is practically no such interval. These aspects of the data comprise a wedge-shaped area of visibility-determined motion thresholds. bounded at the botton by durations exceeding the critical duration and at the top by conditions for which the time-distance trateoff (Eq. 3) provides energy in excess of visibility requirements.
The present empirically derived equations, then, can account for almost all aspects of R. H. Brown's principal 1957 and 1958 data. But these equations can also describe motion threshold under stimulus conditions which Brown's experimental design, because of its use of luminance as the dependent variable. is incapable of measuring. i.e.. conditions under which threshold becomes independent of luminance. as reflected by the vertical asymptotes in Figs. $5 \mathrm{~b}$ and 6 . Moreover. the time-distance tradeoff (expressed by Eq. 3), which applies to the data approaching these asymptotes, is far more generally descriptive of threshold conditions across possible combinations of the relevant stimulus dimensions. and is always so descriptive when the target is not so dim as to permit visibility requirements to limit liminal stimulus relationships.

\section{Temporal Integration and Distance}

One facet of Brown's 1957 data which the preceding treatment has not taken into account is his finding of an increase in the energy criterion for visibility of the target with increases in the distance of its movement. It can be shown that the energy quantities represented by the unit-slope asymptotes of Fig. 5a are slightly greater for greater extents. The same effect in different guise. is reflected in the present author's data in that the so-called "energy constancy limbs" (see Fig. 4) were, in fact. characterized on the average by a slightly greater energy quantits at the second and find point. for which threshold distance was alwar s greater (see Figs. 1 and 3).

These findings reflect the fact that temporal integration is less efficient the greater the retinal distance oree which it must operate (thouth this affect is nealigible at small distances). Tome-luminancis 
reciprocity still applies at the larger distances, but the constant-energy sum yielded is higher (Graham \& Margaria, 1935): that is. temporal integration is complete, but spatial integration is partial. If distance is a free variable, however, as is the case for Fig. 4 . reciprocity will not strictly apply, though it is a good approximation for small distances.

Brown offers no mathematical fit for his data on the interaction of energy and distance, but they seem amenable to description by

$$
L t=e^{c_{1} d+c_{2}}
$$

where $e$ is the base of the naturai iogaritims, $c_{1}$ and $c_{2}$ are constants. and $d$ is the distance of movement. Since simple $\mathrm{Lt}$ reciprocity is a tolerable approximation for the present author's data, it has been used for the sake of simplicity in the preceding treatment and mathematics. To have a generally valid mathematical account, however, it is Eq. 5, rather than Bloch's law, which must be used to describe threshold values of the stimulus variables when motion discrimination is limited by visibility requirements. It is also $\mathrm{Eq} .5$ which must be used to determine the values at which the more generally applicable relationships, expressed by Eq. 3. yield to such visibility requirements and, therefore, to Eq. 5 . Equations 3 and 5 may be used as simultaneous equations when one variable is fixed as a parameter. If Eq. 5 were applied in these ways to the theoretical functions of Fig. 6. it would necessitate a positive acceleration in the curve representing intersections between the linear and nonlinear limbs. This would have the effect of yielding the same decreasing lateral separations between extent functions which is manifest in Brown's data in Fig. 5a and. therefore. the same associated increases in asymptote energy.

\section{Isochronal Results}

This consideration is also important for an analysis of Brown's 1955 study. in which he first reported the finding of energy constancy for movement discrimination. In this study. Brown measured threshold luminance for motion discrimination at each of numerous velocities with duration fixed and repeated such measurements for a number of different durations. He reported that threshold luminance was at a minimum for some single velocity at each duration, and it was these minimum luminances which were used to construct a flat Lt-reciprocity curve of the conventional kind. Superficially, these results are somewhat puzzling, since the data for any given duration do not show energy constancy (i.e.. threshold luminance varies, so the energy product $\mathrm{Lt}$ also varies). while the particular values extracted across different durations do.

Combining the present author's findings with the interaction of distance and absolute threshold energy expressed by Eq. 5. a plot may be constructed showing the predicted behavior of movement threshold given Brown's independent, dependent, and parametric variables. Figure 7 plots theoretical threshold luminance as a function of velocity for each of several exposure durations. The descending portions of each function were generated by the appropriate solution of Eq. 3 and the ascending portions by Eq. 5 . Equation 3 constants are the same as those used in generating Figs. $3 \mathrm{~b}$ and 6; Eq. 5 constants were estimated from the same data. Points of intersection between the two relationships may be determined by solving simultaneous equations for each value of $t$.

It can be seen that each isochronal function, rather than exhibiting Lt constancy, is characterized by a minimum luminance at some intermediate speed within the associated velocity range. It may be assumed that these are the minima found by Brown, for which he demonstrated a reciprocity between time and luminance. Since the points of intersection pick off nearly the same point on each visibility-limited function-and since these functions are relatively flat at this point-these minima represent virtually the same energy content. For the four decreasing $t$ values in Fig. 7 , the associated minimum log $\mathrm{Lt}$ values, for example, are $.65, .66, .67$, and .68 . Thus, these points yield nearly perfect $\mathrm{Lt}$ reciprocity across durations, despite the fact that such reciprocity is not at all characteristic of any single function.

\section{CONCLUSION}

R. H. Brown's motion studies constituted a giant step beyond earlier research in that they were probably the first to break the stimulus down into its fundamental dimensional components and to provide in terms of these components a precise, if restricted, depiction of the basic input requirements of the detection mechanism. In view of the findings of Henderson (1971) and the present analysis of Brown's data, however, it appears that some of his conclusions require additional qualification. Brown credits to movement discrimination as a "visual effect" the Lt reciprocity which the present treatment, and even Brown's data, indicates is merely transmitted to motion threshold by the limiting visibility function. In this light, it seems a misconstruction to assert, as Brown (1958) does, that under certain conditions motion discrimination, "like visibility. requires a minimal amount of energy." The appropriate adjunctive phrase would be "because of visibility"; that is, motion discrimination requires visibility, and visibilit." "requires a minimal amount of energy."

Brown's conclusions about the generality of energy constancy also appear in need of updating. Brown (1957) states without qualification. for example. that "for exposure times shorter than a critical duration, a constant amount of energy $(L t)$ is required for the discrimination" of motion. In fact. energy constancy applies only for durations less than a critical duration and greater than some smaller value for which the 
Fig. 7. Theoretical curves relating threshold luminance for motion discrimination and velocity. with duration as a parameter.

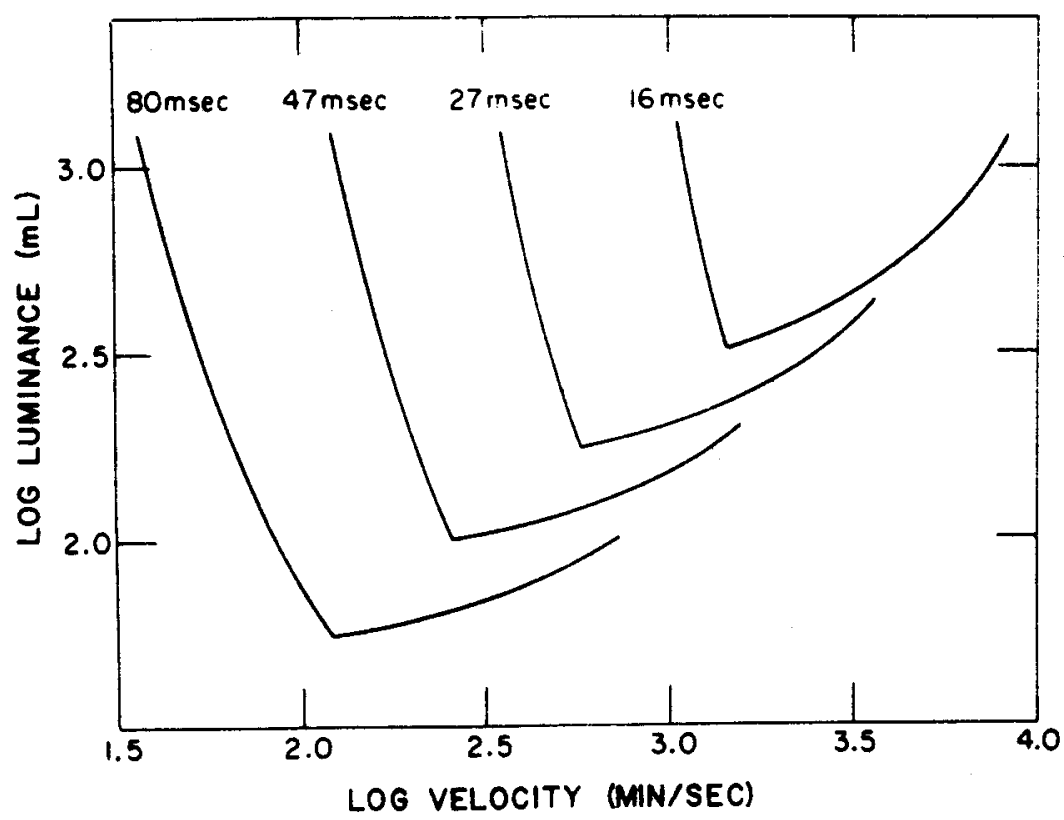

motion and visibility functions diverge. Similarly. Brown`s (1958) statement that energy constancy applies to motion threshold "for moderate speeds" is inappropriately restricted. It can be seen from Brown's own data (Fig. 5a) that whether or not motion threshold at a given velocity falls on the unit-slope reciprocity curve depends on the spatial extent of that motion.

As for the effect of exposure duration. Brown (1957) says. "There is no indication that time is important as a variable other than in providing for the [constant] supply of energy within the time required for the discriminatory event." If this were true. the motion threshold functions (Figs. 5a and 5b) would never depart from the linear reciprocity curves. which represent for each extent combinations of time and luminance which provide a constant energy supply. The fact is that for each extent. threshold durations. decreasing along the unit-slope functions as the result of increasing velocity. reach a critical minimum value bevond which motion threshold luminance veers toward infinity. asymptotic with a constant velocity or duration. This occurs despite the fact that successively shorter durations would continue to provide that same constant amount of energy which is sufficient to sustain threshold at longer durations.

It seems well established. then. that the perceptual event labeled "movement" is not triggered by the retinal accumulation of some critical energy' quantity. At least in this sense. there is no single photochemical sensory event. The data suggest. to the contrary. that movement perception requires some critical separation in space and time between at least wo sensory events. such requirements being less in either dimension given larger separations in the other. These characteristics suggest to the present author the inverse relationship between retinal area and the critical duration at which complete temporal integration breaks down. Whatever the nature of retinal participation in movement discrimination (given the stimulus conditions assumed herein). whether it relies on unspecialized receptor associations or on motion-specific units. the coding process must demand input data according to the relationships which have been found to describe the threshold stimulus.

\section{REFERENCES}

Barlow. H. B., \& Hill. R. M. Selective sensitivity to direction of motion in ganglion cells of the rabbit's retina. Science. 1963. $139.412-414$

Barlow. H. B., Hill. R. M.. \& Levick. W. R. Retinal ganglion cells responding selectively to direction and speed of image motion in the rabbit. Journal of Physiology. 1964, 173, 377-407.

Bouman. M. A..\& van den Brink. G. On the integrate capacity in time and space of the human peripheral retina. Journal of the Optical Society of America. 1952.42.617-620.

Bouman. M. A.. \& van den Brink. G. Absolute thresholds for moving point sources. Journal of the Optical Society of America, $1953,43,895-898$.

Brown. R. H. Velocity discrimination and the intensity-time relation. Journal of the Optical Sociaty of America, 1955.45. 189-192.

Brown. R. H. The effect of extent on the intensity-time relation for the visual discrimination of movement. Journal of Comparative \& Physiological Psychology. 1957. 50. 109-114.

Brown. R. H. Influence of stimulus luminance upon the upper speed threshold for the visual discrimination of movement. Journal of the Optical Society of America. 1958,48, 125-128.

Cohen. R. L.. \& Bonnet. C. Movement detestion thresholds and stimulus duration. Perception \& Psychophysics. 1972. 12. $269-272$

Graham. C. H. \& Margaria. R. Area and the intensity-time relationship in the peripheral retina. American Joumal of Phrsiology. 1935.113. 299-305

Henderson. D. C. The ralationships among time. distance and 
intensity as determinants of motion discrimination.

Perception \& Psychophysics. 1971, 10. 313-320.

Oyster, C. W. The analysis of image motion by the rabbit retina.

Journal of Physiology, 1968. 199.613-635.

Sperling. H. G.. \& Jolliffe. C. L. Intensity-time relationship at threshold for spectral stimuli in human vision. Journal of the Optical Society of America. 1965. 55, 191-199.

(Received for publication May 25, 1972; revision received October 31,1972 .) 